\title{
Modeling Partially Surveyed Point Process Data: Inferring Spatial Point Intensity of Geomagnetic Anomalies
}

\section{Authors: Kenneth A. Flagg, Andrew Hoegh, John J. Borkowski}

This is a post-peer-review, pre-copyedit version of an article published in Journal of Agricultural, Biological and Environmental Statistics. The final authenticated version is available online at: https://doi.org/10.1007/s13253-020-00387-2. The following terms of use apply: https:// www.springer.com/gp/open-access/publication-policies/aam-terms-of-use.

Flagg, Kenneth A., Andrew Hoegh, and John J. Borkowski. "Modeling Partially Surveyed Point Process Data: Inferring Spatial Point Intensity of Geomagnetic Anomalies." Journal of Agricultural, Biological and Environmental Statistics 25, no. 2 (March 12, 2020): 186-205. doi:10.1007/ s13253-020-00387-2. 


\title{
Modeling Partially Surveyed Point Process \\ Data: Inferring Spatial Point Intensity of Geomagnetic Anomalies
}

\author{
Kenneth A. Flagg, Andrew Hoegh, and John J. Borkowski
}

Many former military training sites contain unexploded ordnance (UXO) and require environmental remediation. For the first phase of UXO remediation, locations of geo-magnetic anomalies are recorded over a subregion of the study area to infer the spatial intensity of anomalies and identify high concentration areas. The data resulting from this sampling process contain locations of anomalies across narrow regions that are sur-veyed; however, the surveyed regions only constitute a small proportion of the entire study area. Existing methods for analysis require selecting a window size to transform the partially surveyed point pattern to a point-referenced dataset. To model the partially surveyed point pattern and infer intensity of anomalies at unsurveyed regions, we propose a Bayesian spatial Poisson process model with a Dirichlet process mixture as the inhomogeneous intensity function. A data augmentation step is used to impute anomalies in unsurveyed locations and reconstruct clusters of anomalies that span surveyed and unsurveyed regions. To verify that data augmentation reconstructs the underlying structure of the data, we demonstrate fitting the model to simulated data, using both the full study area and two different sampled subregions. Finally, we fit the model to data collected at the Victorville Precision Bombing range in southern California to estimate the intensity surface in anomalies per acre.

\section{INTRODUCTION}

Across the USA, many sites used by the military for training have been sold to private owners or made available for use by the public. However, these sites contain debris resulting from munitions use activities, often including unexploded ordnance (UXO) which failed to detonate and remain potentially hazardous, and hence, require environmental remediation. 
Remediation projects initially collect preliminary data in narrow regions along a sample of straight-line transects where magnetometers or digital geophysical mapping (DGM) equipment are used to identify locations of metallic items, known as geomagnetic anomalies. This data structure, with locations of anomalies recorded along the transects, is referred to as a partially surveyed point pattern. Using terminology from Cressie (1991), the location of anomalies will be referred to as anomaly events, or just events.

Existing methods for partially surveyed point patterns in the UXO context require transforming the anomaly events from the point pattern into point-referenced data. The contribution of this work is to develop a statistical method to infer an intensity surface across both the sampled and unsampled regions by imputing anomaly events in non-surveyed regions. To model such data with a point process, we introduce a nonparametric procedure where the intensity surface is constructed with a Dirichlet process mixture to model observed anomalies events and impute across unsampled areas to identify regions of high intensity across the entire study region.

\subsection{UXO OVERVIEW}

At terrestrial firing ranges, such as those used to train artillery or bombing crews, geomag-netic anomalies originate from three processes: (i) firing of munitions, (ii) the site geology, and (iii) other human use of the site (small arms use, recreation, etc.). Anomalies of type (i) are expected to build up in the vicinity of targets, resulting in high-intensity regions known as target areas (TAs). There is ballistic justification for TAs to be roughly elliptical, and size estimates based on fragmentation distances of common munitions are available from US Army Corps of Engineers sources. Anomalies of type (ii) are considered background noise and are traditionally assumed to occur at a constant intensity in regions with approximately homogeneous geologic properties. Anomalies of type (i) need to be dug up and removed from the site, but they cannot be differentiated from types (ii) or (iii) until they are dug. Digging is time-consuming and expensive, so the purpose of estimating the intensity surface is to identify the boundaries of potential TAs before any digging occurs.

When the locations of TAs are unknown, to save resources for environmental remediation, surveys are conducted across narrow rectangular areas. The survey is sparse relative to the TA size, with transects placed far enough apart so that as few as two transects could traverse a TA of the smallest size consistent with the type of munitions believed to be present. At the Victorville Precision Bombing Range, the motivating dataset for this article, data were collected in 2-m-wide swaths along transects placed $78 \mathrm{~m}$ apart with the goal of identifying TAs for $100 \mathrm{lb}$ air-to-surface bombs. This surveying plan called for $2.6 \%$ of the total area of the site to be surveyed. The data consist of the locations of detected anomalies.

While anomaly locations are naturally modeled as a partially observed realization of a spatial point process, the standard procedure transforms the anomaly locations into pointreferenced data by computing a moving average of the number of anomalies per unit area. This procedure is available in Visual Sample Plan (Matzke et al. 2014, VSP), the de facto standard software package used by Department of Defense contractors to plan data collection and carry out statistical mapping for ordnance remediation projects. Anomalies per unit area are calculated as the number of detected anomalies in a small circular window divided 
by the area surveyed within the window. The moving average is calculated for windows centered at regular intervals along the transects. In this manner, the list of anomaly locations is converted to a list of numerical values attached to different locations. Then ordinary Kriging methods developed for point-referenced data are applied to predict the moving average intensity across the site. This analysis requires the choice of moving average window size, which introduces arbitrary structure not present in the original data. The window size is a tuning parameter with no relation to the process being modeled, but the estimated covariance parameters and resulting intensity map are sensitive to this choice. Furthermore, the averaging induced by the moving window procedure will end up smoothing the intensity value. Spatial point process models bypass this arbitrary choice and directly describe the locations of the anomalies.

\subsection{Point Process OVerview}

Spatial point processes are random processes whose realizations are sets of discrete events in space, with the observed set of events known as a spatial point pattern (Diggle 2013). Spatial point process models are statistical models which jointly describe the number and spatial configuration of events. Spatial point process models tend to be computationally intense to fit (Møller and Waagepetersen 2003), but many likelihood-based methods for fitting these models are available in the spatstat package (Baddeley and Turner 2005; Baddeley et al. 2015) for R (R Core Team 2018).

There are two common ways to represent the distribution of a point process. The classical representation is the Radon-Nikodym derivative of the probability function with respect to a standardized Poisson process; this derivative in referred to as the density with respect to a Poisson process. An alternate representation that is useful for Bayesian models is a hierarchical construction created by conditioning on the number of events (Taddy et al. 2012). The result resembles the joint distribution of the number of events and their distribution over space. This article uses the latter representation, with $f$ denoting the probability density function of an event (the spatial domain being the support).

Spatial point process methods have occasionally been applied to munitions data. MacDonald and Small (2006) use $K$-functions to study the clustering structure of munitions at Army firing ranges. Cressie and Lawson (2000) fit a superposition model to events identified from photographs of a minefield to classify the events as either mines or background noise. The entire site was surveyed in each of these applications; we are not aware of any previous applications of point process methods to partially surveyed UXO sites.

There is precedent for model-based extrapolation in the spatial point process literature. Van Lieshout and Baddeley (2002) discuss extrapolation of cluster processes beyond the observed region, mentioning mine clearance as a possible application. Brix and Møller (2001) fit a spatiotemporal log-Gaussian Cox process model to the locations of weeds photographed in rectangular plots during an agricultural study and mapped the estimated intensity across the entire field. Waagepetersen and Schweder (2006) and Møller and Waagepetersen (2007) use a shot noise Cox process to analyze a Minke whale dataset with a partially surveyed structure, but the goal of these analyses is to estimate whale abundance over the region rather than localized intensity. Ji et al. (2009) fit a Poisson process 
model driven by a Dirichlet process mixture of multivariate Gaussians to immunofluorescence images, using block MCMC sampling to impute imperfectly observed pixel-level presence/absence. More recently, Gabriel et al. (2016) derive connections between geosta-tistical processes and spatial point processes, modeling the point intensity of Montagu's harrier nests from a stationary geostatistical process using an ordinary Kriging method to predict the intensity in an unsurveyed region; this Kriging procedure is further developed in Gabriel et al. (2017).

Our goal is to construct a spatial point process model for geostatistical anomalies at UXO sites and then develop an approach for fitting this model in situations where the study area is only partially observed. The current approach for modeling UXO events, as in VSP, requires coercing the anomaly events to point-referenced data and then using a Kriging procedure. There are potential issues with treating events that arise from a point process in this approach; in particular, mapping the data to point-referenced data requires an arbitrary window size selection that can over smooth the data. Some of the methods described in the previous section, such as the log-Gaussian Cox model and the Gabriel et al. (2016, 2017) procedure, can be applied to the partially observed point pattern; however, both assume the intensity function is driven by single process at work across the whole region and emphasize estimating its covariance function. Furthermore, neither of these methods directly imputes events outside the surveyed regions. In contrast, we define a procedure that imputes anomaly events and the intensity function as a mixture model of munitions fired at discrete targets.

We propose a Bayesian spatial Poisson process model with an intensity function designed to model TAs. The number and locations of potential TAs are typically unknown, so the intensity function is modeled as an infinite mixture of bivariate Gaussian kernels. We place a Dirichlet process prior (Antoniak 1974; Teh 2011) on the mixing distribution. A data augmentation procedure accounts for possible point configurations in the unsurveyed portion of the site. The desired outcome of this analysis is an intensity map across the entire study area, including both the surveyed and non-surveyed regions, as a precursor to identifying TAs and to inform remediation decisions.

While this paper focuses on analyzing UXO events, the methods presented here are widely applicable. In addition to the partially observed point pattern scenarios already described, transect sampling is common in environmental and ecological settings (Burnham et al. 1980). With transect sampling, the research question traditionally focuses on estimating a population total across the study area. However, in many situations spatial inferences concerning locations of events are also of interest, but until recently methods for point process data were not applicable for partially observed sampling approaches. The modeling approach presented in this work provides an approach for estimating population totals across the region of interest, but also creating maps with spatial intensity.

This paper is organized as follows. Section 2 introduces the Victorville Precision Bombing Range site used as a motivating case study. Section 3 presents the model and explains how to fit the model using a Gibbs sampling algorithm. Section 4 demonstrates fitting the model to simulated data while Sect. 5 presents analysis of data from the Victorville Precision Bombing Range near Victorville, CA. Section 6 concludes with discussion and future research directions. 


\section{MOTIVATING DATASET: VICTORVILLE PRECISION BOMBING RANGE}

The Victorville Precision Bombing Range, southeast of Victorville, California, was previously studied as part of a Wide Area Assessment Project of the Department of Defense's Environmental Security Technology Certification Program (Hathaway et al. 2007). One goal of the Wide Area Assessment was to demonstrate the application of statistical mapping tools to data collected along transects using vehicle-towed DGM arrays. The study site is a 5500-acre section of the bombing range where 100-lb bomb exercises were known to have taken place in a valley between two mountainous ridges. These activities were expected to produce elliptical TAs at least $500 \mathrm{ft}$ (152 m) along each axis. Visible craters marked the presence of a target at the center of the site. Records also indicated another target existed somewhere in the southeastern section of the site despite an absence of craters.

Anomaly events were collected using a vehicle-towed DGM device with a $2 \mathrm{~m}$ width. To be conservative, the sampling plan was based upon the smallest TA size expected, a circle 152 $\mathrm{m}$ in diameter. Transects were placed $78.1 \mathrm{~m}$ apart with a goal of having a 0.99 probability of traversing and detecting a TA of that size, as calculated in VSP. The original sampling plan called for transects running east to west across the site. If this plan was applied to the whole site, the survey would have covered $2.6 \%$ of the site area. However, rocky terrain prevented the towed DGM equipment from accessing some sections of the site, so only $1.5 \%$ of the site was surveyed. After the first round of data collection, the analysis team requested that additional data be collected to better estimate the local anomaly intensity in four observed high-intensity regions near the edges of the site and in the cratered region in the center. The additional data were collected along short north-to-south transects, and the combined surveys along east-west and north-south transects covered $1.6 \%$ of the site area.

The objective is to make inferences about the intensity function of a point process across the entire 5500-acre Victorville site to identify TAs using information obtained from a surveyed region over which the detection equipment passed. The site is a bounded two-dimensional region denoted $R$, and the surveyed region is a subset $S$ and is also two-dimensional. In practice, the data collection crew had to navigate around unexpected obsta-cles and difficult terrain, so the actual path traversed does not exactly match the planned transects. Therefore, $S$ was defined from GPS data recorded during data collection and not from the sampling plan.

The complete data consist of the Cartesian coordinates of 1900 detected anomalies, comprising the elements of the set $\mathbf{X}^{(S)}$, and a sequence of waypoints recording the path traversed by the detection equipment, called the Course Over Ground and used to construct a representation of $S$ (Fig. 1). To construct $S$, the waypoints were first connected in sequence by line segments, producing a one-dimensional path. The path was then expanded into a twodimensional polygon encompassing the region that would have been underneath the detector (which has a known width) assuming the equipment traveled around the site while staying centered on the path. The quality of this representation depends upon the resolution and frequency with which the waypoints were recorded, but the focus of this paper is on the 


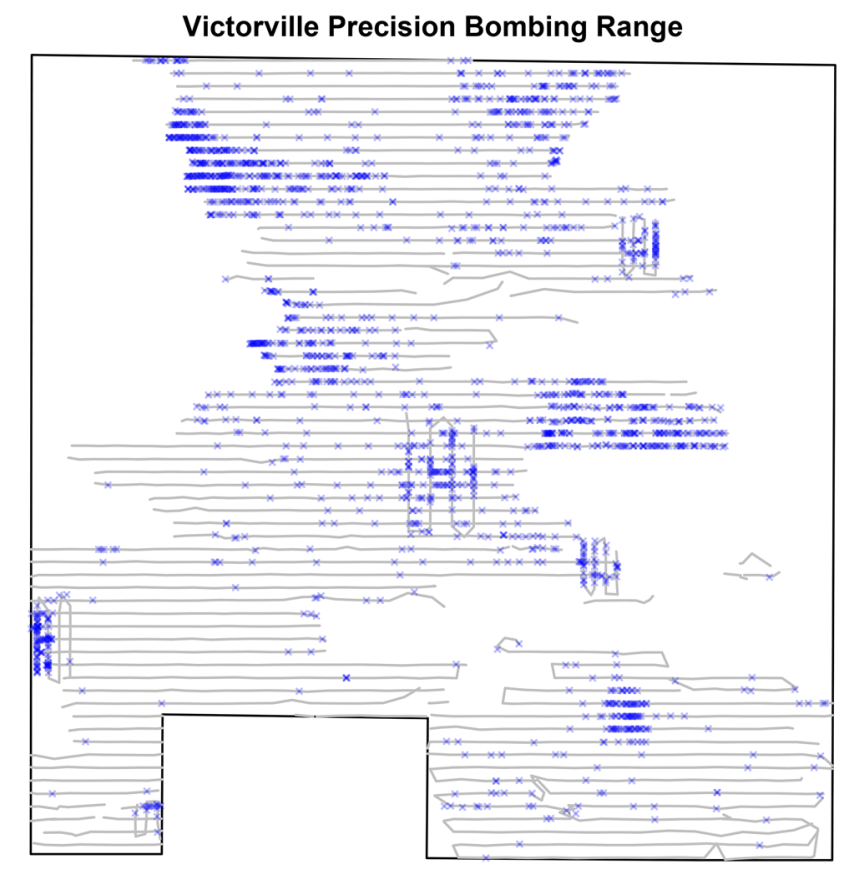

Figure 1. Map of the Victorville site showing the defined region of interest (black outline), surveyed section (gray lines) and the detected geomagnetic anomalies (blue points).

point process model, so we assume that this construction of $S$ is accurate enough that any errors will not affect the results.

\section{MODEL}

The novelty in our application is the use of data augmentation to explore the possible distributions of anomalies in the unobserved region of the spatial point process. We model the locations of geomagnetic anomalies as a realization of a spatial point process, which will be used to predict regions representing possible TAs over the entire site, including non-surveyed areas. By design, only a subset of the region of interest was surveyed. We assume the boundaries of the observed region are known exactly. Augmenting the recorded anomalies with latent anomaly locations allows the model to predict TAs that extend beyond the boundaries of the observed region. We assume that the site contains $n$ munitions-related items resulting from explosive weaponry fired at targets, with the munitions impacting (and typically fragmenting) in the vicinity of each target. The resulting geomagnetic anomalies correspond to the intact munitions and fragmented debris. Anomalies are expected to be most concentrated near the target with the intensity tapering off farther away, and may reasonably be modeled using a Dirichlet Process mixture model composed of bivariate Gaussian distributions.

A Dirichlet process mixture model (DPMM) provides a mechanism for fitting an infinite mixture model where the number of mixture components and the associated weights do not 
need to be prespecified. With the Dirichlet process mixture model, data points, which in the UXO case correspond to anomaly events, are either assigned to an existing mixture com-ponent or create a new mixture component. For a comprehensive overview of the Dirichlet process, readers are referred to Teh (2010). Formally, the Dirichlet process mixture model can be written in a hierarchical manner where:

$$
\begin{aligned}
\mathbf{x}_{i} \mid \boldsymbol{\theta}_{i} & =F\left(\boldsymbol{\theta}_{i}\right) \\
\boldsymbol{\theta}_{i} \mid G & \sim G \\
G \mid \alpha, G_{0} & \sim D P\left(\alpha, G_{0}\right)
\end{aligned}
$$

$\boldsymbol{x}_{\mathbf{1}}, \ldots, \boldsymbol{x}_{\boldsymbol{n}}$ are a set of events, $\boldsymbol{\theta}_{1}, \ldots, \boldsymbol{\theta}_{n}$ are latent parameters drawn from the distribution $G$. Due to the nature of the Dirichlet process, $\mathrm{G}$ is a discrete distribution so the $\boldsymbol{\theta}_{i}$ values are not necessarily unique. Rather the $\boldsymbol{\theta}_{i}$ values correspond to the cluster parameters: the mixture component centers $\left(\boldsymbol{\mu}_{l}\right)$ and the mixture component covariance matrices $\left(\boldsymbol{\Sigma}_{l}\right)$. Hence, $F\left(\boldsymbol{\theta}_{i}\right)$ is a two-dimensional Gaussian distribution with parameters distribution $\boldsymbol{\mu}_{l}$ and $\boldsymbol{\Sigma}_{l}$.

We define a Bayesian inhomogeneous Poisson process model by factoring the intensity function into the mean number of events and the probability density function of the locations of events across the study region. In the model, $n$ follows a Poisson distribution with mean $\Lambda$. Conditional on the mixing distribution, the intensity function is

$$
\lambda(\mathbf{x} \mid \Lambda, G)=\Lambda f(\mathbf{x} \mid G),
$$

where $f(\mathbf{x} \mid G)$ is the probability density function of events across $\mathcal{R}$. This has the form of an infinite mixture, namely

$$
f(\mathbf{x} \mid G)=\int \mathrm{N}_{2}(\mathbf{x} \mid \boldsymbol{\mu}, \boldsymbol{\Sigma}) \mathrm{d} G(\boldsymbol{\mu}, \boldsymbol{\Sigma})
$$

where $\mathrm{N}_{2}$ is the bivariate normal density function. Let

$$
\mathbf{X}=\left\{\mathbf{x}_{1}, \ldots, \mathbf{x}_{n}\right\}
$$

be the set of all events in $\mathcal{R}$. Note that the assigned indices are arbitrary, but we will consider them fixed for the sake of providing clear notation. The distribution of $\mathbf{X}$ is

$$
[\mathbf{X} \mid \Lambda, G]=\frac{\exp (-\Lambda)}{n !} \prod_{\mathbf{x} \in \mathbf{X}} \Lambda f(\mathbf{x} \mid G)
$$

where an empty product is taken to be 1 (Cressie 1991).

The expression above describes a fully surveyed site where all events are observed and their locations recorded. We need a model for the process $\mathbf{X}^{(\mathcal{S})}$ in the observed region $\mathcal{S}$ and the process $\mathbf{X}^{(U)}$ in the unobserved region $\mathcal{U}=\mathcal{R} \backslash \mathcal{S}$. A property of the Poisson process is that, conditional on the intensity function, the processes in disjoint regions are independent Poisson processes. That is, the joint distribution of $\mathbf{X}^{(\mathcal{S})}$ and $\mathbf{X}^{(\mathcal{U})}$ is 


$$
\left[\mathbf{X}^{(\mathcal{S})}, \mathbf{X}^{(\mathcal{U})} \mid \Lambda, G\right]=\frac{\exp \left(-\Lambda^{(\mathcal{S})}\right)}{n^{(\mathcal{S}) !}}\left(\prod_{\mathbf{x} \in \mathbf{X}^{(\mathcal{S})}} \Lambda f(\mathbf{x} \mid G)\right) \times \frac{\exp \left(-\Lambda^{(\mathcal{U})}\right)}{n^{(\mathcal{U}) !}}\left(\prod_{\mathbf{x} \in \mathbf{X}^{(\mathcal{U})}} \Lambda f(\mathbf{x} \mid G)\right)
$$

where $\Lambda^{(\mathcal{S})}=\Lambda \int_{\mathcal{S}} f(\mathbf{x} \mid G)$ and $\Lambda^{(\mathcal{U})}=\Lambda \int_{\mathcal{U}} f(\mathbf{x} \mid G)$.

We complete the Bayesian model by specifying prior distributions. We chose conditionally conjugate forms for priors to facilitate Gibbs sampling: $\Lambda \sim \operatorname{Gamma}(a, b)$, and for the mixing distribution, we use the $\operatorname{DP}\left(\alpha, G_{0}\right)$ Dirichlet process prior, setting $G_{0}(\boldsymbol{\mu}, \boldsymbol{\Sigma})$ to specify independent $\boldsymbol{\mu} \sim \mathrm{N}(\boldsymbol{\eta}, \boldsymbol{\Psi})$ and $\boldsymbol{\Sigma} \sim \operatorname{InvWishart}(\nu, \boldsymbol{\Omega})$.

The complete model specification is

$$
\begin{aligned}
n \mid \Lambda, G & \sim \operatorname{Poisson}(\Lambda), \\
\mathbf{x}_{i} \mid G & \sim f\left(\mathbf{x}_{i} \mid G\right) \text { for } i=1, \ldots, n, \\
\Lambda & \sim \operatorname{Gamma}(a, b), \\
G & \sim \operatorname{DP}\left(\alpha, G_{0}\right)
\end{aligned}
$$

where $\alpha, \boldsymbol{\eta}, \boldsymbol{\Psi}, v, \boldsymbol{\Omega}, a$, and $b$ are hyperparameters to be specified.

Ideally, the subject matter expertise would be used to inform both the spacing of the transects in the initial sampling design and the prior distributions of model parameters. In particular, $\boldsymbol{\Sigma}$ corresponds to the size of the mixture components used to create the intensity surface. The posterior intensity function is sensitive to $\boldsymbol{\Omega}$. This parameter sets the scale of the mixture components, which is directly related to their size. If the scale is too small, the result is many small mixture components with few anomaly events in each. If the scale is excessively large, all of the anomaly events may be assigned to single component that poorly describes the structure of the site. An $\boldsymbol{\Omega}$ consistent with the a priori expected TA size seems to be a reasonable starting point.

The model is fit using Gibbs sampling, alternatively simulating a realization of each random variable from its complete conditional distribution. The anomaly locations in the unobserved region $\mathcal{U}$ are included among these random quantities. At each iteration, the algorithm produces a possible realization of the point process in the unsurveyed region, which is then used to update the complete conditional distribution of $G$. This allows the sampling algorithm to more fully explore the posterior distribution of the intensity than what could be inferred from the anomaly locations in $\mathcal{S}$ alone. A detailed derivation of the sampling algorithm appears in the supplementary materials.

After initialization, the sampling algorithm proceeds by iterating the following steps:

1. Assign new mixture component labels $k_{i}, i=1,2, \ldots, n$, from

$$
\begin{aligned}
& \operatorname{Pr}\left(k_{i}=l \mid \mathbf{X},\left\{k_{i^{\prime}}: i^{\prime} \neq i\right\},\left\{\boldsymbol{\mu}_{l}\right\},\left\{\boldsymbol{\Sigma}_{l}\right\}\right) \\
& \quad= \begin{cases}\frac{h_{l}^{(-i)}}{\alpha+n-1} \mathrm{~N}_{2}\left(\mathbf{x}_{i} ; \boldsymbol{\mu}_{l}, \boldsymbol{\Sigma}_{l}\right) & l=1,2, \ldots, m^{(-i)} \\
\frac{\alpha}{\alpha+n-1} \int \mathrm{N}_{2}\left(\mathbf{x}_{i} ; \boldsymbol{\mu}, \boldsymbol{\Sigma}\right) \mathrm{d} G_{0}(\boldsymbol{\mu}, \boldsymbol{\Sigma}) & l=m^{(-i)}+1\end{cases}
\end{aligned}
$$

The integral evaluates to the multivariate $t$ density (Carvalho et al. 2010). $h_{l}^{(-i)}$ is the number of points in the $l$ th component, not counting the $i$ th point, and $m^{(-i)}$ is the 
number of mixture components that contain any points other than the $i$ th point. That is, $m^{(-i)}=m-1$ if point $i$ was assigned to a singleton component in the previous iteration, and otherwise $m^{(-i)}=m$. After assigning the labels, update $m$ and $h_{l}$, $l=1, \ldots, m$.

2. Draw new centers $\boldsymbol{\mu}_{l}, l=1,2, \ldots, m$, from

$$
\boldsymbol{\mu}_{l} \mid \mathbf{X},\left\{k_{i}\right\}, \boldsymbol{\Sigma}_{l}, \boldsymbol{\eta}, \boldsymbol{\Psi} \sim \mathrm{N}_{2}\left(\boldsymbol{\eta}_{l} . \boldsymbol{\Psi}_{l}\right)
$$

where

$$
\boldsymbol{\Psi}_{l}=\left(\boldsymbol{\Psi}^{-1}+h_{l} \boldsymbol{\Sigma}_{l}^{-1}\right)^{-1}
$$

and

$$
\boldsymbol{\eta}_{l}=\boldsymbol{\Psi}_{l}\left(\boldsymbol{\Psi}^{-1} \boldsymbol{\eta}+\boldsymbol{\Sigma}_{l}^{-1} \sum_{i: k_{i}=l} \mathbf{x}_{i}\right)
$$

3. Draw new covariances $\boldsymbol{\Sigma}_{l}, l=1,2, \ldots, m$, from

$$
\boldsymbol{\Sigma}_{l} \mid \mathbf{X},\left\{k_{i}\right\}, \boldsymbol{\mu}_{l}, v, \boldsymbol{\Omega} \sim \operatorname{invWishart}\left(v+h_{l}, \boldsymbol{\Omega}_{l}^{-1}\right)
$$

with

$$
\boldsymbol{\Omega}_{l}=\boldsymbol{\Omega}+\sum_{i: k_{i}=l}\left(\mathbf{x}_{i}-\boldsymbol{\mu}_{l}\right)\left(\mathbf{x}_{i}-\boldsymbol{\mu}_{l}\right)^{T}
$$

4. Update $c^{(\mathcal{S})}=\int_{\mathcal{S}} f(\mathbf{x} \mid G) \mathrm{d} \mathbf{x}$ and $c^{(\mathcal{U})}=\int_{\mathcal{U}} f(\mathbf{x} \mid G) \mathrm{d} \mathbf{x}$ (by Monte Carlo integration or another numerical method).

5. Draw $\Lambda$ from $\Lambda \mid \mathbf{X}^{(\mathcal{S})}, G \sim \operatorname{Gamma}\left(a+n^{(\mathcal{S})}, b+c^{(\mathcal{S})}\right)$.

6. Draw $n^{(\mathcal{U})}$ from $n^{(\mathcal{U})} \mid \Lambda, G \sim \operatorname{Poisson}\left(c^{(\mathcal{U})} \Lambda\right)$ and update $n=n^{(\mathcal{S})}+n^{(\mathcal{U})}$.

7. Draw mixture component labels $k_{i}, i=n^{(\mathcal{S})}+1, \ldots, n$ from

$$
\operatorname{Pr}\left(k_{i}=l\right)= \begin{cases}\frac{h_{l}}{\alpha+n} & l=1,2, \ldots, m \\ \frac{\alpha}{\alpha+n} & l=m+1\end{cases}
$$

where $h_{l}$ is the number of points in the $l$ th component.

8. Simulate locations $\mathbf{x}_{i}, i=n^{(\mathcal{S})}+1, \ldots, n$ from

$$
\mathbf{x}_{i} \mid k_{i}, \boldsymbol{\mu}_{k_{i}}, \boldsymbol{\Sigma}_{k_{i}} \sim \begin{cases}\mathrm{N}_{2}\left(\boldsymbol{\mu}_{k_{i}}, \boldsymbol{\Sigma}_{k_{i}}\right) & k_{i}=1,2, \ldots, m \\ G_{0}(\boldsymbol{\mu}, \boldsymbol{\Sigma}) & k_{i}=m+1\end{cases}
$$

and update $\mathbf{X}=\mathbf{X}^{(\mathcal{S})} \cup \mathbf{X}^{(\mathcal{U})}$. 
We implemented the Gibbs sampling algorithm in the $\mathrm{R}$ and $\mathrm{C}++$ programming languages using the Armadillo linear algebra library (Sanderson and Curtin 2016) and the RcppArmadillo package (Eddelbuettel and Sanderson 2014) for R.

\section{ARTIFICIAL DATA EXAMPLE}

To demonstrate the modeling framework, an artificial data example is created to mimic the UXO setting where the data are analyzed where the spacing between the transects is progressively increased. The dataset is a realization of a Poisson process with the following assumptions: The study region $\mathcal{R}$ is the square region $[-1.5,1.5] \times[-1.5,1.5]$; the number of anomalies is drawn from the Poisson distribution with mean $\Lambda=200$; and the anomaly locations are distributed as a mixture of two bivariate Gaussian components (Table 1). This results in an intensity function with two hot spots representing TAs. Our objectives for this analysis are to assess the accuracy of the posterior intensity function and to investigate whether the mixture components themselves can be used to delineate high-intensity regions.

The model was fit to the complete realization of the point process (simulating a complete survey of the site area) and to two subsets of the anomalies assuming surveying plans varying the area covered (Table 2). For both plans, the regions surveyed are rectangles each centered over a straight-line transect running north-south over the whole length of the site. The transects were selected by a systematic random sample with a randomly selected starting point and equal space between adjacent transects. The dense survey plan covers half of the site area. The sparse plan has transects spaced widely enough that at most two transects can cross the smaller of the two TAs. When considering sparsity of the sampling approach in our scenario, the important comparison is the spacing of the transects relative to the expected width of the TAs rather than total proportion of the study region that is sampled. Having two transects that cross the TA is comparable to the sparsity of real-world UXO surveys. The surveyed region and observed anomaly locations from each survey plan appear in Fig. 2.

The prior distribution parameter values we selected were $\alpha=1, \eta=(0,0)^{T}, \Psi=\mathbf{I}_{2}$ (the $2 \times 2$ identity matrix), $v=4, \boldsymbol{\Omega}=0.04 \mathbf{I}_{2}, a=0.001$, and $b=0.001$. Preliminary experimentation with the model suggested that the smoothness of the posterior intensity function was sensitive to the choice of $\boldsymbol{\Omega}$. We chose $\boldsymbol{\Omega}$ to imply circular components with prior standard deviation of 0.2 in both directions to represent an expert's prior guess at the

Table 1. Characteristics of the true intensity function associated with the two TAs at the artificial site

\begin{tabular}{|c|c|c|c|c|c|}
\hline Mixing weight & Center & Covariance & Major SD & Orientation & Minor SD \\
\hline 0.375 & $\left(\begin{array}{c}-0.5 \\
0.5\end{array}\right)$ & $\left(\begin{array}{cc}0.0125 & -0.015 \\
-0.015 & 0.0425\end{array}\right)$ & 0.221 & $0.625 \pi$ & 0.079 \\
\hline 0.625 & $\left(\begin{array}{c}0.5 \\
-0.5\end{array}\right)$ & $\left(\begin{array}{cc}0.09 & 0 \\
0 & 0.0225\end{array}\right)$ & 0.3 & 0 & 0.15 \\
\hline
\end{tabular}

The mixing weight is the probability that a realized anomaly comes from each TA. Eigen decomposition of each covariance matrix yields the standard deviation (scale) along both the major and minor axes as well as the orientation of the major axis 
Table 2. Descriptions of the survey plans applied to the simulated site

\begin{tabular}{llllll}
\hline \hline Plan & Description & Width & Spacing & Area observed & Anomalies observed \\
\hline Fully surveyed & Full site & - & - & 9 units $^{2}$ & 179 \\
Densely surveyed & North-south transects & 0.05 & 0.10 & 4.5 units $^{2}$ & 97 \\
Sparsely surveyed & North-south transects & 0.05 & 0.30 & 1.5 units $^{2}$ & 36 \\
\hline
\end{tabular}

The "Width" column gives the width of the strip observed along each transect (i.e., the footprint of the detection equipment) and the "Spacing" column gives the distance between adjacent transects (i.e., the distance between the center lines of adjacent rectangles
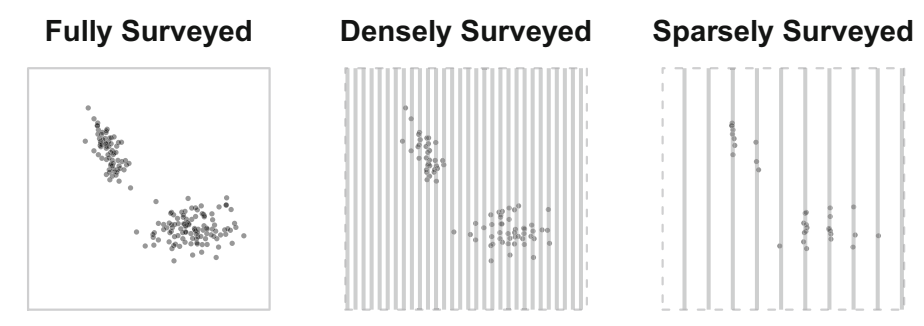

Figure 2. The anomaly locations (black circles) at the simulated site (left) and as observed under two different survey plans (center and right). All of these anomalies represent munitions debris; real sites typically have background noise as well. The area of the site observed under each sampling plan is shaded in grey.

TA size. We chose $\boldsymbol{\eta}$ to center the distribution of mixture component centers on the middle of the site. Our choice of $\boldsymbol{\Psi}$ gives this distribution a standard deviation of 1 in either direction, allowing the centers to be disbursed across the $3 \times 3$ square or even outside of it. We selected the other parameters to yield weak prior distributions.

For the data observed under each survey plan, six Gibbs sampling chains were initialized from the prior distribution and run in parallel for 50,000 iterations. For the fully surveyed dataset, data augmentation was not needed so steps 6-8 of the algorithm were omitted. For the densely and sparsely surveyed data, the full algorithm was used. Convergence was assessed by viewing trace plots of the number of mixture components $m$ and by examining plots showing the mixtures for individual iterations. The number of components started out very large because some early iterations assigned most of the anomalies to their own components, but this number dropped to less than 20 components within the first 100 iterations for all three datasets.

\subsection{Fully Surveyed Site Results}

Unsurprisingly, the model fit to the fully observed data yielded results close to the parameters used to generate the data. The posterior distribution of $\Lambda$ was centered on the observed number of anomalies (median of 179), and the central 95\% posterior interval was 153.9206.9. The posterior number of mixture components $m$ had a median of 3 and central $95 \%$ interval of 2-8.

The posterior mean intensity had two hot spots around the observed anomalies, with an elevated mean intensity between the TAs (Fig. 3a, left). However, these hot spots tended 
Posterior Mean Intensity
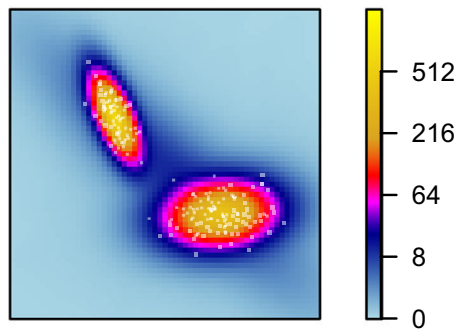

Posterior Intensity SD

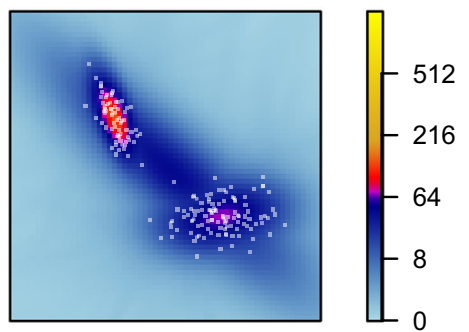

(a) Fully Surveyed

Posterior Mean Intensity

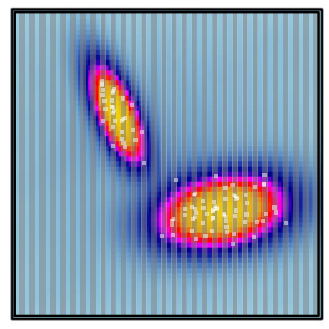

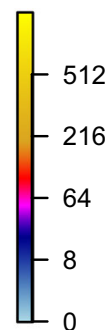

Posterior Intensity SD
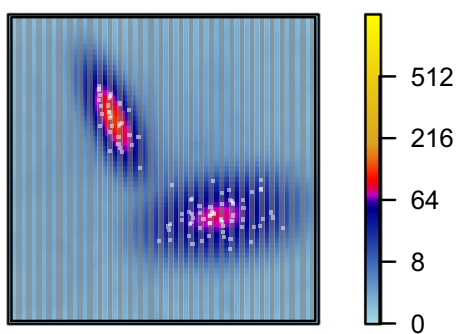

(b) Densely Surveyed

\section{Posterior Mean Intensity}

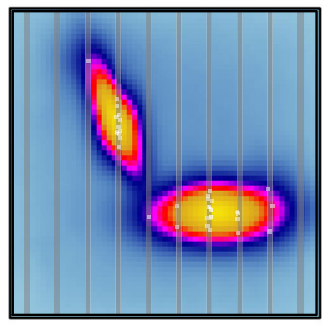

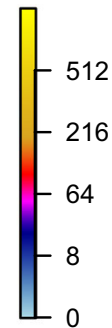

Posterior Intensity SD
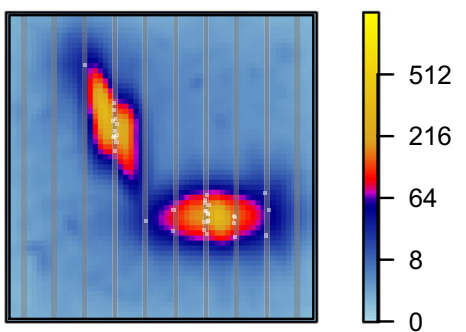

(c) Sparsely Surveyed

Figure 3. Posterior mean and standard deviation of $\lambda(\mathbf{x})$ obtained using the fully surveyed data. The intensity function was calculated on a $61 \times 61$ lattice. The observed anomalies appear as small white dots.

to be constructed from more than two mixture components and the posterior probability of $m=2$ was only 0.235 . Often, some anomalies from each TA were assigned to spatially large mixture components covering parts of both TAs. Most iterations included two components corresponding to the two visually apparent clusters of anomalies, but when additional components were present they included long, narrow components running northwest to southeast across the site and covering both clusters of anomalies. The standard deviation 
of the intensity function showed evidence of these additional components (Fig. 3a, right). However, these components were generally of low weight. Compared to other regions where no anomalies were observed, the standard deviation was larger in the section between the two high-intensity regions to reflect the uncertainty in the presence of diagonal mixture component (Fig. 3a).

\subsection{Densely Surveyed Site Results}

The results of fitting the model to the data from the first survey plan (covering half of the site) included additional variability in the posterior, but otherwise resembled the results obtained when using the fully surveyed site. The posterior median of $\Lambda$ was 193.5 , and the central 95\% posterior interval for was 157.0-234.5. The posterior distribution of $m$ had a median of 4 and a central $95 \%$ posterior interval from 2 to 7 . Again, most posterior draws included components that matched the two clusters of anomalies.

In the unsurveyed area, anomalies are occasionally predicted far from the observed anomalies. These anomalies are then assigned to new components which have low mixing probabilities. As a result, the posterior probability of $m=2$ was 0.132 . Mixture components with large area covering both TA regions were uncommon. The posterior mean intensity function looked similar to that resulting from the fully surveyed site.

\subsection{Sparse Survey Results}

The data from the sparse survey had some interesting characteristics due to the large spacing between transects. Discrete "stacks" of anomalies along the transects were apparent, as shown in Fig. 2, and all but one observed anomaly from the northwest TA were on one transect that went through the center of the TA. Ideally, we would hope to see the data augmentation procedure fill in the space between adjacent transects with predicted anomaly locations, reconstructing the TAs.

The results from fitting the model to this dataset showed considerable posterior uncertainty due to the low proportion of the area surveyed. The posterior distribution of $\Lambda$ had a median of 209.3 and a long right tail, with the central $95 \%$ posterior interval extending from 144.5 to 308.8 . The posterior distribution of $m$ also had a long right tail, with a median of 6 , a central $95 \%$ posterior interval of 3-10, and a maximum of 16 mixture components.

The plot of the posterior mean intensity surface (Fig. 3c, left) looked similar to the plots created using the other datasets. The primary difference was that, in this case, the highintensity region in the upper left is somewhat diamond shaped and tended to be made up of several overlapping mixture components. The posterior probability that $m=2$ was only 0.044 . The posterior standard deviation was very large, especially between transects inside the TAs (Fig. 3c, right). This was due to variability in the number and locations of predicted anomalies, which led to variation in the mixture components to which they were assigned. The data augmentation procedure did successfully repopulate the TAs with anomalies. 


\subsection{SUMMARY}

Our novel point process model was able to construct an accurate intensity function even with relatively low sampling effort. The posterior variability in the mean number of anomalies, number of mixture components, and intensity increased with sparsity of the surveying plan, as expected. Sparser sampling corresponded to larger numbers of mixture components. We observed two explanations for this. First, anomalies predicted in the data augmentation step spawned "unobserved" components consisting mostly of latent anomalies located between the observed rectangles. Second, the elliptical shapes were less apparent from the observed anomaly locations, so more components were necessary to describe the observed data with more complicated shapes. The individual posterior mixture components in general did not correspond to entire TAs, but rather the mixture results in a smooth surface that accurately reflects the true intensity surface.

The data augmentation process proved necessary in the artificial data scenario. If the data augmentation steps were omitted with the partially surveyed datasets, long skinny clusters form along the sampled regions. Using the data augmentation process to impute events results in fairly accurate reconstructions of the underlying intensity surface.

The sparsity of the sampling procedure impacts both the results from model fitting procedure as well as the computational effort required. If the sampling approach is too sparse, or in other words if the spacing between the sampling regions is too large relative to the width of the TAs, TAs may not be identified from the sampled data. This would lead to numerical instability and inconsistent model output. As is generally good practice, care should be taken in the sample design phase. Given data recorded at the sampling regions, the overall sparsity of the sampled region does have computational implications. The data augmentation step is the most computationally intensive part of the model fitting procedure; hence, the trade-off with sparser sampling plans is that additional computing time is required.

Our choice of prior parameter values allowed the data to dominate. The observed anomaly locations defined the centers and covariances of the mixture components, with little influence from the priors. Overall, the Gibbs sampling algorithm performed satisfactorily in finding the posterior distribution of the intensity function using data simulated from a simple model.

A standard approach for model diagnostics with point process models is to compare the fitted model with a process exhibiting complete spatial randomness. Given the scenario we are studying, this comparison is unnecessary as the simulated data and real dataset clearly exhibit heterogeneity. Additionally, model fit diagnostics for point process models using residual diagnostics are popular (Ogata 1988; Baddeley et al. 2005, 2011, 2013). The basic idea of these diagnostics is to define a set $\mathcal{B}$, which is a small spatial region, for which the residual is calculated as the difference between the expected number of points and observed number of points. With the posterior means in Fig. 3a, the models do a nice job of reconstructing the intensity surface used to simulate the data. However, with a partially surveyed study area, residual diagnostics do not exist for the non-surveyed areas; however, formal diagnostics or visual inspection can be conducted for observed areas. In Sect. 5, residual figures are constructed for the observed path in the Victorville dataset; however, residual diagnostics for partially surveyed point process data is an area for future research. 


\section{ANALYSIS: VICTORVILLE PRECISION BOMBING RANGE}

In this section, we return to the Victorville Precision Bombing Range site, described in Sect. 2. This is a 5500-acre site where 100-lb bomb use was expected to produce TAs 152 $\mathrm{m}$ in diameter. To identify TAs, $1.6 \%$ of the site area was surveyed in 2-m-wide strips along transects. We set the prior parameter values to reflect the TA size prior information used to create the surveying plan while being uninformative in other respects. The prior distribution of the mixture component covariances had $v=4$ and $\boldsymbol{\Omega}=(152 / 4)^{2} \mathbf{I}_{2}$, representing circular target areas with diameters of roughly $152 \mathrm{~m}$. This is informed by subject matter expertise about the TAs. The subject matter expertise should ideally be used to construct the initially sampling plans too. The target area centers were distributed with mean $\eta$ set to the centroid of the site. The site fits inside a $4885 \mathrm{~m}$ by $4898 \mathrm{~m}$ window, so the covariance $\boldsymbol{\Psi}$ was defined as a diagonal matrix selected with entries $1221.3^{2}$ and $1224.5^{2}$. Together, these choices of $\eta$ and $\boldsymbol{\Psi}$ imply that mixture components are most likely to be spatially centered in the middle of the site but still allow the flexibility for components to be centered in the outskirts or even outside of the site. The remaining parameters were $\alpha=1, a=0.001$, and $b=0.001$, selected to be uninformative.

We ran the Gibbs sampling algorithm of Sect. 3 with six chains for 50,000 iterations each. Convergence was assessed by examining trace plots of the parameters and by studying plots showing the mixtures for a random sample of iterations. The trace plots all suggested that convergence was achieved around 20,000 iterations, so the first 20,000 iterations were discarded as burn-in. The mixture plots showed that all chains routinely captured the same high-intensity features while other small mixture components appeared and disappeared quickly and without any apparent pattern. This implies good mixing of the chains. Due to computer memory constraints, we only retained every fiftieth iteration, resulting in a final sample of 3600 draws from the posterior.

The intensity surface is most clearly described through the posterior distribution of the intensity function. Figure 4 shows the posterior mean (top) and standard deviation (bottom) of the intensity function calculated on a $201 \times 201$ lattice. In the plot of the posterior mean intensity, the magenta color corresponds to 53 anomalies per acre (ApA), the threshold used in Hathaway et al. (2007) to delineate high-intensity regions. The plot shows many regions of varying shapes and sizes where the posterior mean intensity exceeded $53 \mathrm{ApA}$. The data augmentation procedure connects recorded anomalies across transects with several small and circular regions appearing where a small number of anomalies were clustered away from other areas of high intensity. Of the larger high-intensity regions, two have the elliptical shape expected of TAs (in the upper right and lower right of the plot) while others have more complicated shapes. Several high-intensity regions extend beyond the vicinity of the transects rather than conforming to the shape of the surveyed section of the site. However, the posterior mean intensity drops to zero far beyond the transects, so this map should not be used to extrapolate a large distance outside of the surveyed portion of the site. For purposes of this analysis, extrapolation is restricted to areas within $40 \mathrm{~m}$ of an area that was sampled. Given the transect spacing was about $78 \mathrm{~m}$, this would include areas between transects as well as additional area that surrounds the boundaries of the transects. 

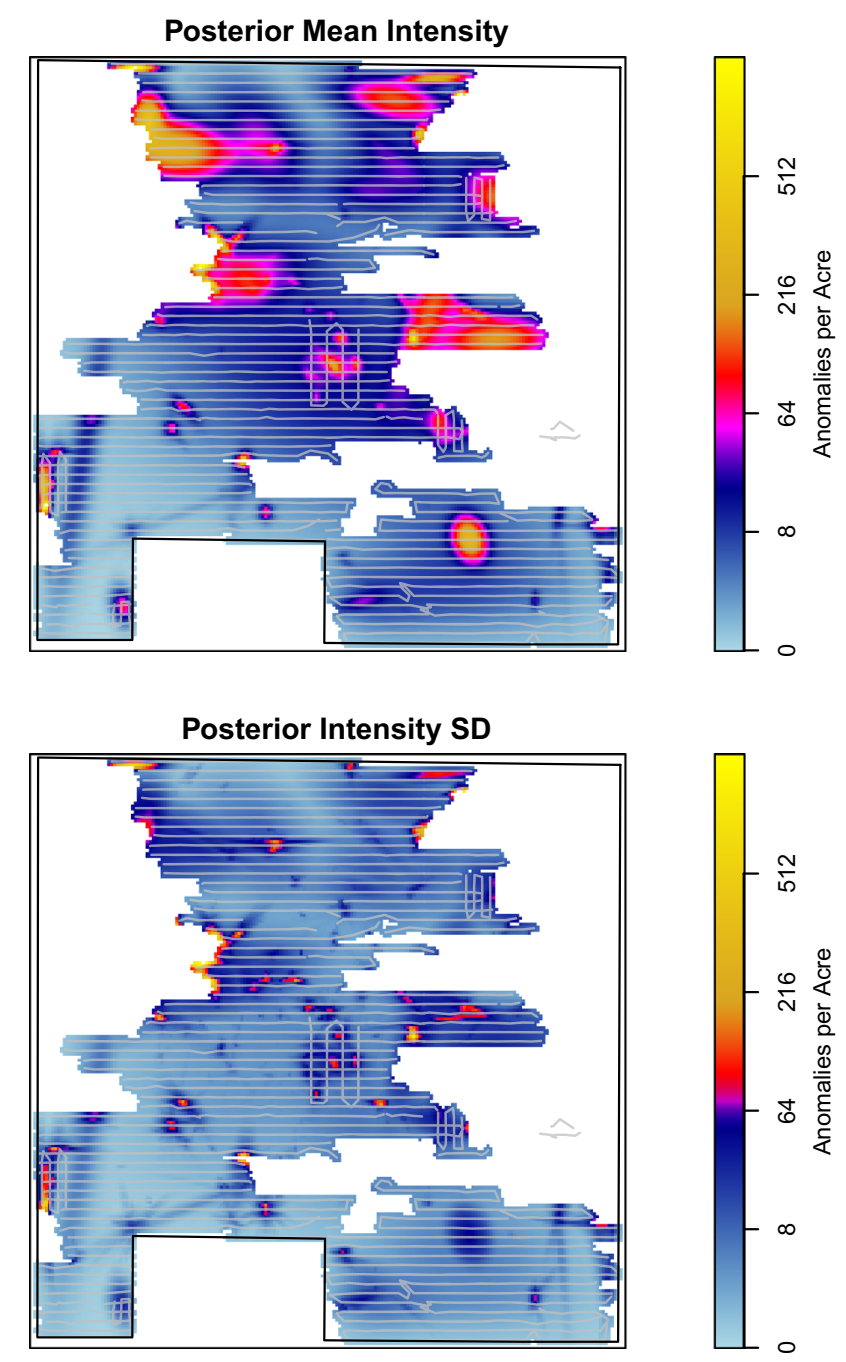

Figure 4. Posterior mean and standard deviation (SD) of anomaly intensity, in anomalies per acre (ApA), for the Victorville Precision Bombing Range. The surveyed region appears as a lightly shaded overlay.

While residual diagnostics cannot be conducted for the entire study area, they can be applied for the observed path. Figure 5a contains Pearson residuals for our model using the data augmentation procedure to impute points. The lack of spatial structure is encouraging. Furthermore, we also examined residuals without using the imputation procedure in Fig. $5 \mathrm{~b}$; however, the figure contained residuals of a larger magnitude, that tended to be positive, and had spatial structure, which implies the data imputation procedure is necessary.

The posterior mean intensity function appeared generally similar to the surface obtained from the moving average Kriging procedure presented in the Wide Area Assessment, with most intensity values in the same range of 0-300 ApA and with the same regions showing elevated intensity. However, the posterior mean intensity surface is rougher, with many small regions of very high intensity. Near the edges of the site, some of these high-intensity hot 


\section{COG-Segment Pearson Residuals}

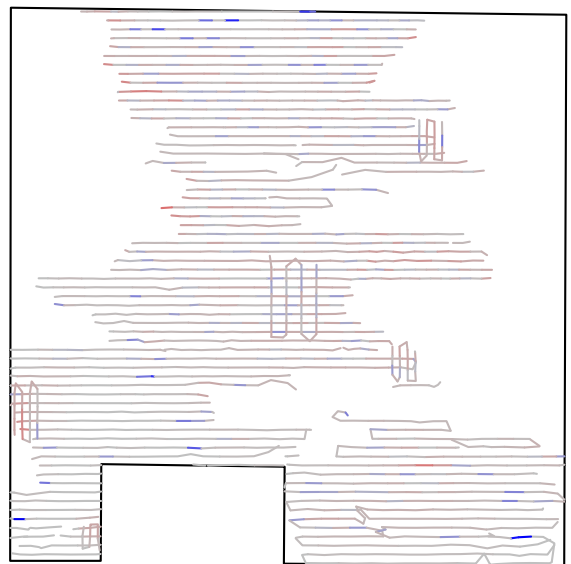

(a) Intensity surface from DPMM using data augmentation to impute events in non-surveyed regions.

COG-Segment Pearson Residuals

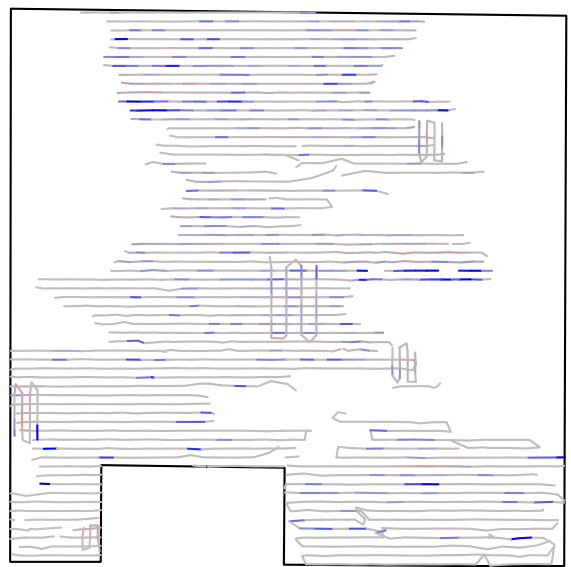

(b) Intensity surface from DPMM without using data augmentation to impute events in non-surveyed regions.

Figure 5. Pearson residuals for the Course Over Ground in the Victorville dataset. Both figures are on the same scale where dark blue corresponds to large positive residuals and dark red corresponds to large negative residuals.

spots approach extreme values near 800 ApA. These appear because the DP mixture allows the components to vary in size to fit the data well; no such regions are visible in Figure 21 of Hathaway et al. (2007) due to the fixed $300 \mathrm{~m}$ window diameter.

Although the resulting intensity maps are similar, our Bayesian spatial point process model has conceptual and interpretation advantages over the moving average Kriging method. The Kriging method interpolates between derived quantities with no assumed structure or relation to the data-generating model. In contrast, the model introduced in this paper 
incorporates elliptical structures consistent with the ballistics of explosive munitions. And while the Kriging approach requires the choice of the window size (a fixed smoothing parameter unrelated to the data-generating process), our Bayesian model replaces this choice with a prior specification that can be tied more closely to the expected TA size. As a model more closely resembling the data-generating process, the Bayesian spatial point process model promises more rich inference than previous methods.

\section{DISCUSSION}

Standard methods to model partially surveyed UXO data treat the data as point-referenced and require specifying a moving average window size. There are some existing methods for partially observed point pattern data; however, none are explicitly designed for the clustering data structure of UXO events. Additionally, these existing methods do not provide a mechanism for imputing anomaly events in non-surveyed regions. To fill this need, a point process model, using a Dirichlet process, is introduced to augment the data with latent anomaly locations across the non-surveyed regions. The point process model and associated data augmentation step successfully fill in anomalies between transects to accurately reconstruct the intensity function. For the sparsely surveyed artificial dataset, anomalies from one true TA were observed only along one transect except for a single anomaly along an adjacent transect. Notably, this TA was oriented at an angle to the transects. The data augmentation procedure placed the stack of anomalies observed along one transect, the lone anomaly on the next transect, and several latent anomalies between the two transects into the same mixture component. As a result, the posterior intensity function supported a high-intensity region of roughly the same location, size, and orientation as the true TA.

Finally, while our proposed model is effective at mapping anomaly intensity, it comes with a heavy computational burden. Our Gibbs sampling procedure takes at least several days to run on the real-world dataset used. The greatest difficulty is in integrating $f$ in every iteration, with a large number of lattice points or Monte Carlo integration draws needed to approximate the integral when the area of $\mathcal{S}$ is small relative to $\mathcal{R}$. Our ongoing work incorporates approximate inference and high-performance computing to improve the tractability of fitting complex point process models to data from partially surveyed sites.

The posterior distribution tended to include more mixture components than the true number of TAs present at the simulated site even though they were truly bivariate normal mixture components. This difference does not prevent the method from accurately recreating the data-generating intensity function, but it indicates that individual mixture components should not be taken to represent TAs. The flexibility of the Dirichlet process will stand out in fitting the intensity surface with overlapping components helping to model more complicated shapes. When irregularly shaped high-intensity regions are present, a large number of small components would be beneficial. We should expect the posterior number of components to be large, but the intensity function will capture the shapes of the high-intensity regions and help to identify potential TAs.

While the application here is targeted on UXO remediation, partially surveyed point processes occur in many applications in the agricultural, biological, and environmental 
sciences, particularly when transect sampling is employed. Our method also provides a way to ask spatially referenced research questions from partially observed point pattern data that arise from transect sampling. Building on the novel advances in this article, our future work combines adaptive sampling techniques for integrated sampling and estimation of partially observed point pattern data.

\section{ACKNOWLEDGEMENTS}

Our many thanks go to John Hathaway for providing the Victorville Precision Bombing Range data. We also thank Megan Higgs for her guidance and mentorship throughout the research process, and for reviewing drafts of this manuscript with her fine attention to detail. Finally, we thank Kate Catlett and Neptune \& Company, Inc., for asking tough questions about UXO remediation and providing for the application. This research topic would not have come to our attention were it not for Neptune striving for the highest possible standards of statistical rigor in their work.

\section{REFERENCES}

Antoniak, C. E. (1974), 'Mixtures of Dirichlet processes with applications to Bayesian nonparametric problems', The Annals of Statistics pp. 1152-1174.

Baddeley, A., Chang, Y.-M. and Song, Y. (2013), 'Leverage and influence diagnostics for spatial point processes',

Scandinavian Journal of Statistics 40(1), 86-104.

Baddeley, A., Rubak, E., Møller, J. et al. (2011), 'Score, pseudo-score and residual diagnostics for spatial point process models', Statistical Science 26(4), 613-646.

Baddeley, A., Rubak, E. and Turner, R. (2015), Spatial Point Patterns: Methodology and Applications with R, Chapman and Hall/CRC Press.

Baddeley, A. and Turner, R. (2005), 'Spatstat: An R package for analyzing spatial point patterns', Journal of Statistical Software 12(6), 1-42.

Baddeley, A., Turner, R., Møller, J. and Hazelton, M.(2005), 'Residual analysis for spatial point processes (with discussion)', Journal of the Royal Statistical Society: Series B (Statistical Methodology) 67(5), 617-666.

Brix, A. and Møller, J. (2001), 'Space-time multi type log Gaussian Cox processes with a view to modelling weeds', Scandinavian Journal of Statistics 28(3), 471-488.

Burnham, K. P., Anderson, D. R. and Laake, J. L. (1980), 'Estimation of density from line transect sampling of biological populations', Wildlife monographs (72), 3-202.

Carvalho, C. M., Lopes, H. F., Polson, N. G., Taddy, M. A. et al.(2010), 'Particle learning for general mixtures', Bayesian Analysis 5(4), 709-740.

Cressie, N. (1991), Statistics for Spatial Data, Wiley.

Cressie, N. and Lawson, A. B. (2000), 'Hierarchical probability models and Bayesian analysis of mine locations', Advances in Applied Probability 32(2), 315-330.

Diggle, P. (2013), Statistical Analysis of Spatial and Spatio-Temporal Point Patterns, 3rd edn, CRC Press.

Eddelbuettel, D. and Sanderson, C. (2014), 'Rcpparmadillo: Accelerating R with high-performance c++ linear algebra', Computational Statistics and Data Analysis 71, 1054-1063.

Gabriel, E., Bonneu, F., Monestiez, P. and Chadœuf, J.(2016), 'Adapted Kriging to predict the intensity of partially observed point process data', Spatial Statistics 18, 54-71.

Gabriel, E., Coville, J. and Chadœuf, J. (2017), 'Estimating the intensity function of spatial point processes outside the observation window', Spatial Statistics 22, 225-239. 
Hathaway, J. R., McKenna, S. and Pulsipher, B. (2007), Application of statistically based site characterization tools to Victorville Precision Bombing Ranges Y and 15 for the ESTCP wide area assessment demonstration, Technical report, Environmental Security Technology Certification Program. https://www.serdp-estcp.org/ content/download/5978/81382/file/MM-0325-Victorville.pdf

Ji, C., Merl, D., Kepler, T. B. and West, M. (2009), 'Spatial mixture modelling for unobserved point processes: Examples in immunofluorescence histology', Bayesian Analysis 4(2), 297.

MacDonald, J. A. and Small, M. J. (2006), 'Assessing sites contaminated with unexploded ordnance: statistical modeling of ordnance spatial distribution', Environmental Science \& Technology 40(3), 931-938.

Matzke, B., Wilson, J., Newburn, L., Dowson, S., Hathaway, J., Sego, L., Bramer, L. and Pulsipher, B. (2014), Visual Sample Plan Version 7.0 User's Guide, Pacific Northwest National Laboratory, Richland, Washington. http://vsp.pnnl.gov/docs/PNNL-23211.pdf

Møller, J. and Waagepetersen, R. (2007), 'Modern spatial point process modelling and inference', Scandinavian Journal of Statistics 34, 643-711.

Møller, J. and Waagepetersen, R. P. (2003), Statistical Inference and Simulation for Spatial Point Processes, CRC Press.

Ogata, Y. (1988), 'Statistical models for earthquake occurrences and residual analysis for point processes', Journal of the American Statistical Association 83(401), 9-27.

R Core Team (2018), R: A Language and Environment for Statistical Computing, R Foundation for Statistical Computing, Vienna, Austria. https://www.R-project.org/

Sanderson, C. and Curtin, R. (2016), 'Armadillo: a template-based c++ library for linear algebra', Journal of Open Source Software.

Taddy, M. A., Kottas, A. et al. (2012), 'Mixture modeling for marked poisson processes', Bayesian Analysis 7(2), 335-362.

Teh, Y. W. (2010), 'Dirichlet process', Encyclopedia of machine learning pp. 280-287.

Teh, Y. W. (2011), Dirichlet process, in 'Encyclopedia of Machine Learning', Springer, pp. 280-287.

Van Lieshout, M. and Baddeley, A. (2002), Extrapolating and interpolating spatial patterns, in A. Lawson and D. Denison, eds, 'Spatial Cluster Modelling', CRC Press, chapter 4, pp. 61-86.

Waagepetersen, R. and Schweder, T. (2006), 'Likelihood-based inference for clustered line transect data', Journal of Agricultural, Biological, and Environmental Statistics 11(3), 264. 